\title{
ALGEBRAIC DIFFERENCE BETWEEN $p$-CLASSES OF AN H*-ALGEBRA
}

\author{
LAJOS MOLNÁR \\ (Communicated by Palle E. T. Jorgensen )
}

\begin{abstract}
We show that there do not exist surjective ring homomorphisms between different $p$-classes of an infinite-dimensional $\mathrm{H}^{*}$-algebra.
\end{abstract}

\section{INTRODUCTION AND PRELIMINARIES}

It is well known that not every subspace of a Banach space $X$ is the range of a bounded linear operator on $X$. The problem of describing the subspaces of $X$ which are so-called operator ranges and which are not was studied in several papers (see [5] and the references therein). Now it seems natural to consider the similar question for homomorphisms (continuity is not assumed). The aim of this note is to present a class of examples for subalgebras of particular but important algebras which are not homomorphism ranges. We reach our goal of proving that the $p$-classes of an $\mathrm{H}^{*}$ algebra as rings differ from each other. These spaces can be considered as common generalizations of $p$-classes of compact operators and $l_{p}$ spaces (cf. $[9,11,21,22]$ ). This difference exists in spite of the fact that the methods for constructing them are rather the same. Although the involved structures are algebras, we consider them only as rings and every algebraic concept (homomorphism, isomorphism, etc.) is used in this respect. The approach to dealing with algebras as rings and gaining statements similar to those which would follow if they were treated as algebras has a long history. Besides the probable first work of this kind [3], we refer to some recent results $[18,19,20]$.

In the proof we apply purely algebraic as well as functional analytical techniques, with the deepest results due to Šemrl and Omladic $[12,20]$.

If $H$ is a Hilbert space and $1 \leq p<\infty$, then let $B L(H)$ and $C L_{p}(H)$ denote the set of bounded and $p$-class operators on $H$, respectively.

Throughout the paper $\mathcal{A}$ stands for a semi-simple $\mathrm{H}^{*}$-algebra (i.e. $\mathcal{A}$ is a semisimple Banach *-algebra whose norm is a Hilbert space norm such that $\left\langle x, y z^{*}\right\rangle=$ $\langle x z, y\rangle=\left\langle z, x^{*} y\right\rangle$ for every $\left.x, y, z \in \mathcal{A}\right)$.

Saworotnow discovered that for an $\mathrm{H}^{*}$-algebra the centralizers play the same role as the bounded linear operators do in the case of a Hilbert space. A (left) centralizer is a function $T: \mathcal{A} \rightarrow \mathcal{A}$ such that $T(x y)=(T x) y$ holds for every $x, y \in \mathcal{A}$. In

Received by the editors July 25, 1994 .

1991 Mathematics Subject Classification. Primary 46K15, 47D50; Secondary 46L40.

Key words and phrases. $\mathrm{H}^{*}$-algebra, $p$-classes, Jordan homomorphism.

Research partially supported by the Hungarian National Research Science Foundation, Operating Grant Number OTKA 1652 and K\&H Bank Ltd., Universitas Foundation. 
their excellent paper [15] Saworotnow and Giellis showed that these mappings are automatically continuous linear operators and that they form a $\mathrm{C}^{*}$-subalgebra of $B L(\mathcal{A})$. Moreover, in a series of papers $[13,14,16,17]$ (see the references in the survey [16] as well), Saworotnow developed a theory of centralizers and, more generally, $A$-linear operators on a Hilbert $A$-module which shows many features similar to the classical operator theory on Hilbert space.

In our recent paper [11] generalizing the results of [10], we brought these two theories closer to each other, realizing algebra isomorphisms between some ideals of the centralizer algebra and those of certain direct sums of operator algebras acting on Hilbert spaces. To be more detailed, we first established a spectral theorem for the elements of $C(\mathcal{A})$, the $\mathrm{C}^{*}$-subalgebra of the centralizer algebra generated by the left multiplications. Roughly speaking, the elements of $C(\mathcal{A})$ are the "compact operators" on $\mathcal{A}$. After this we defined $C_{p}(\mathcal{A})$, the $p$-class of $\mathcal{A}$, in a way very similar to that which is commonly done concerning the von Neumann-Schatten $p$-class of compact operators (cf. [21, 22]). To obtain the mentioned ismorphisms between these $p$-classes and certain operator ideals we need the following construction.

Let $I \neq \emptyset$ be a set, and suppose that $\mathcal{A}_{i}$ is a Banach ${ }^{*}$-algebra and $k_{i}$ is a positive real number for every $i \in I$. If $1 \leq p<\infty$, then let

$$
l_{p}\left(\mathcal{A}_{i}, k_{i}, I\right)=\left\{\left(x_{i}\right)_{i \in I}: x_{i} \in \mathcal{A}_{i}(i \in I), \quad\left(\sum_{i} k_{i}\left\|x_{i}\right\|^{p}\right)^{1 / p}<\infty\right\} .
$$

It is easy to see that these spaces (called the $l_{p}$-direct sum of $\left\{\mathcal{A}_{i}\right\}_{i \in I}$ ) with the norms suggested in their definitions are Banach *-algebras.

Now, our cited result, which is strongly used in our present investigations, is the following [11, Theorem 3]:

Let $1 \leq p<\infty$. There exists an isometric *-isomorphism from the Banach *-algebra $C_{p}(\mathcal{A})$ onto $l_{p}\left(C L_{p}\left(H_{\alpha}\right), c_{\alpha}, \Gamma\right)$.

\section{The MAIN RESUlT}

Our theorem treats the non-existence of Jordan homomorphisms between different $p$-classes of $\mathcal{A}$. If $\mathcal{R}_{1}$ and $\mathcal{R}_{2}$ are rings, then the additive function $\Phi: \mathcal{R}_{1} \rightarrow \mathcal{R}_{2}$ is called a Jordan homomorphism if

$$
\Phi\left(x^{2}\right)=\Phi(x)^{2} \quad\left(x \in \mathcal{R}_{1}\right) .
$$

This important notion, which is obviously more general than that of a homomorphism, was introduced by Jacobson and Rickart [7].

Theorem. Let $\mathcal{A}$ be an infinite-dimensional $H^{*}$-algebra. If $1 \leq p, q<\infty, p \neq$ $q$, then there does not exist a surjective Jordan homomorphism from $C_{p}(\mathcal{A})$ onto $C_{q}(\mathcal{A})$.

Proof. The proof is divided into several steps. First observe that by the structure theory of $\mathrm{H}^{*}$-algebras [2], $\mathcal{A}$ is of infinite dimension if and only if either it has an infinite-dimensional minimal closed ideal or there are infinitely many minimal closed ideals each of which is finite dimensional.

Now suppose $\Phi: C_{p}(\mathcal{A}) \rightarrow C_{q}(\mathcal{A})$ is a Jordan homomorphism. We note that by [11, Theorem 3] mentioned above we may and do consider $\Phi$ acting on $l_{p}\left(C L_{p}\left(H_{\alpha}\right), c_{\alpha}, \Gamma\right)$ and let $\Phi_{\alpha}$ denote its $\alpha$ th coordinate function. 
Step A. $\Phi_{\alpha}$ is a homomorphism or an anti-homomorphism.

Since $C L_{p}\left(H_{\alpha}\right)$ is a prime ring, this follows from the classical theorem of Herstein [6, Theorem 3.1].

In what follows assume that $\Phi_{\alpha}$ is a homomorphism. Let $\mathcal{I}$ denote the kernel of $\Phi_{\alpha}$ and for every $\beta \in \Gamma$ let $\mathcal{I}_{\beta}$ stand for the natural projection of $\mathcal{I}$ onto the $\beta$ th coordinate space of $l_{p}\left(C L_{p}\left(H_{\alpha}\right), c_{\alpha}, \Gamma\right)$.

Step B. $\mathcal{I}$ is closed and $\mathcal{I}_{\beta}=\{0\}$ or $\mathcal{I}_{\beta}=C L_{p}\left(H_{\beta}\right)$.

To the closedness of $\mathcal{I}$ we note that by $[11$, Theorem 6$]$ and $[4$, Proposition 8 , p. 223], every $p$-class of a semi-simple $\mathrm{H}^{*}$-algebra is semi-simple. Now the assertion follows from the fact that the kernel of a surjective homomorphism from a Banach algebra onto a semi-simple normed algebra is closed. The proof of this statement can be carried out by following the proof of [4, Proposition 10, p. 131], where linearity is not used. For an arbitrary $x_{\beta} \in C L_{p}\left(H_{\beta}\right)$ let $\overline{x_{\beta}}$ denote the element of $l_{p}\left(C L_{p}\left(H_{\alpha}\right), c_{\alpha}, \Gamma\right)$ whose coordinates are 0 except for the $\beta$ th one which is $x_{\beta}$. Suppose $0 \neq x_{\beta} \in \mathcal{I}_{\beta}$. Since $\mathcal{I}$ is an ideal, we obtain for any $a_{\beta}, b_{\beta} \in C L_{p}\left(H_{\beta}\right)$ that $\overline{a_{\beta} x_{\beta} b_{\beta}} \in \mathcal{I}$. Consequently, for every finite rank operator $f_{\beta}$ on $H_{\beta}$ we have $\overline{f_{\beta}} \in \mathcal{I}$. But we know that $\mathcal{I}$ is closed and the finite rank operators form a dense subspace in $C L_{p}\left(H_{\beta}\right)$. Hence $\overline{x_{\beta}} \in \mathcal{I}$ for any $x_{\beta} \in C L_{p}\left(H_{\beta}\right)$.

Step C. There is exactly one index $\beta$ for which $\mathcal{I}_{\beta}=\{0\}$ holds.

Assume that there are two such indexes, say $\beta_{1}$ and $\beta_{2}$. Consider the well-defined mapping

$$
\Phi_{\alpha}(x) \mapsto\left(\begin{array}{c}
x_{\beta_{1}} \\
x_{\beta_{2}}
\end{array}\right) .
$$

It is a surjective homomorphism from $C L_{q}\left(H_{\alpha}\right)$ onto the direct sum $C L_{p}\left(H_{\beta_{1}}\right) \oplus$ $C L_{p}\left(H_{\beta_{2}}\right)$. If we compose this mapping by the natural projection

$$
\pi_{1}: C L_{p}\left(H_{\beta_{1}}\right) \oplus C L_{p}\left(H_{\beta_{2}}\right) \rightarrow C L_{p}\left(H_{\beta_{1}}\right),
$$

then using [4, Proposition 10, p. 131] again, we would obtain that its kernel is a non-trivial closed ideal in $C L_{q}\left(H_{\alpha}\right)$, which is a contradiction.

If there does not exist an index $\beta$ for which $\mathcal{I}_{\beta}=\{0\}$, then what we have shown in the proof of Step B together with the closedness of $\mathcal{I}$ imply $\mathcal{I}=\mathcal{A}$, but this is untenable.

Let $\beta$ denote the index for which $\mathcal{I}_{\beta}=\{0\}$. Then we have $\mathcal{I}=\left\{x \in \mathcal{A}: x_{\beta}=0\right\}$, and this implies that there exists an isomorphism $\phi_{\alpha}: C L_{p}\left(H_{\beta}\right) \rightarrow C L_{q}\left(H_{\alpha}\right)$ such that

$$
\Phi_{\alpha}(x)=\phi_{\alpha}\left(x_{\beta}\right) \quad(x \in \mathcal{A}) .
$$

Step D. If $H, K$ are Hilbert spaces and $H$ is infinite dimensional, then there does not exist a ring isomorphism from $C L_{p}(H)$ onto $C L_{q}(K)$.

Suppose, conversely, that $\Psi: C L_{p}(H) \rightarrow C L_{q}(K)$ is an isomorphism. By [20, Theorem] $\Psi$ is spatially implemented, i.e. there is an invertible linear or conjugatelinear operator $a: H \rightarrow K$ such that $\Psi(x)=a x a^{-1}$. A contradiction can be reached through the following line of substeps.

Lemma 1. If $H, K$ are Hilbert spaces such that there is an invertible bounded linear or conjugate-linear operator $A: H \rightarrow K$, then $H$ and $K$ are isomorphic as Hilbert spaces.

We deal only with the case in which $A$ is conjugate-linear. Consider the positive linear operator $A^{*} A: H \rightarrow H$, where the adjoint of $A$ (which is bounded 
and conjugate-linear) is denoted by the same symbol as that of a bounded linear operator. Let $P: H \rightarrow H$ denote its positive square-root. Since $A$ is invertible, one can show rather easily that $A^{*} A$ and hence $P$ are also invertible. Now if we define $U: H \rightarrow K$ by $U(P x)=A x(x \in H)$, then $U$ is a conjugate-linear isometry onto $K$. Using polarization, it follows that $\langle U x, U y\rangle=\langle y, x\rangle$ for every $x, y \in H$. This fact together with the surjectivity of $U$ imply that $U$ maps a complete orthonormal system of $H$ into a complete orthonormal system of $K$.

From these observations it follows that in the rest of the proof of our Step D we may and do suppose $H=K$.

Lemma 2. Let $H$ be a Hilbert space, $X \in C L_{p}(H)$. Assume that $A, B: H \rightarrow H$ are bounded either linear or conjugate-linear operators. Then $A X B \in C L_{p}(H)$.

The assertion is well known for linear operators; consequently, we deal only with the case in which $A, B$ are conjugate-linear. First consider the positive bounded linear operator

$$
(A X B)^{*}(A X B)=B^{*}\left(X^{*} A^{*} A X\right) B .
$$

$A^{*} A$ is a positive bounded linear operator, hence there is a positive bounded linear operator $T$ such that $A^{*} A=T^{*} T$. Since $(T X)^{*}(T X)$ is a compact linear operator, there exist a sequence $\left(\lambda_{n}\right)$ of nonnegative real numbers and a sequence $\left(\varphi_{n}\right)$ of orthonormal vectors in $H$ such that

$$
(T X)^{*}(T X)=\sum_{n} \lambda_{n} \varphi_{n} \otimes \varphi_{n} .
$$

Let $S: H \rightarrow H$ be a bounded linear operator for which $S^{*} \varphi_{n}=B^{*} \varphi_{n}$ holds for every $n \in \mathbb{N}$. Then for any $x, y \in H$ we have

$$
\begin{aligned}
& \left\langle B^{*}(T X)^{*}(T X) B x, y\right\rangle=\left\langle B y,(T X)^{*}(T X) B x\right\rangle=\sum_{n} \lambda_{n}\left\langle\varphi_{n}, B x\right\rangle\left\langle B y, \varphi_{n}\right\rangle \\
& =\sum_{n} \lambda_{n}\left\langle x, B^{*} \varphi_{n}\right\rangle\left\langle B^{*} \varphi_{n}, y\right\rangle=\sum_{n} \lambda_{n}\left\langle x, S^{*} \varphi_{n}\right\rangle\left\langle S^{*} \varphi_{n}, y\right\rangle \\
& =\left\langle\sum_{n} \lambda_{n} S^{*} \varphi_{n} \otimes S^{*} \varphi_{n} x, y\right\rangle=\left\langle S^{*}(T X)^{*}(T X) S x, y\right\rangle .
\end{aligned}
$$

Consequently, there are bounded linear operators $T, S$ such that $|A X B|=|T X S|$, from which we obtain the statement.

Now let us turn to complete the proof of Step D. If $p<q$, then there is a $y \in C L_{q}(H) \backslash C L_{p}(H)$. In the case $y=a x a^{-1}$ for some $x \in C L_{p}(H)$ by Lemma 2 we arrive at a contradiction. If $p>q$, the proof is similar.

Since the case when $\Phi_{\alpha}$ is an anti-homomorphism can be treated by simply considering the homomorphism $\Psi_{\alpha}(x)=\Phi_{\alpha}\left(x^{*}\right)(x \in \mathcal{A})$, it follows that the proof is complete if there is an infinite-dimensional minimal closed ideal in $\mathcal{A}$. In the rest of the proof assume that $\mathcal{A}$ has infinitely many minimal closed ideals, each of which is finite dimensional.

Lemma 3. If $\Psi: M_{n \times n} \rightarrow M_{m \times m}$ is a ring isomorphism, then $\Psi$ preserves rankone idempotents and their linear spans. Moreover, $n=m$, and a similar statement holds for ring anti-homomorphisms.

Indeed, $p \in M_{n \times n}$ is an idempotent if and only if $\Psi(p)$ is an idempotent element of $M_{m \times m}$. If $p$ is rank one and $\Psi(p)$ is not rank one, then there exist non-zero 
idempotents $q_{1}, q_{2} \in M_{m \times m}$ such that $\Psi(p)=q_{1}+q_{2}$. But this implies that $p$ is a sum of two non-zero idempotents, which is a contradiction. Since $\Psi$ is an isomorphism, the identity of $M_{m \times m}$ can be written as a sum of rank-one idempotents $q_{1}, \ldots, q_{n}$. Taking traces on both sides of the equation

$$
I=q_{1}+\ldots+q_{n},
$$

we infer $m=n$. Let $p \in M_{n \times n}$ be a rank-one idempotent and $0 \neq \lambda$ be a complex number. We have to show that there is a $\mu \in \mathbb{C}$ such that $\Psi(\lambda p)=\mu \Psi(p)$. If $x \in$ $M_{n \times n}$, then $x \Psi(p)=0$ if and only if $\Psi^{-1}(x) \lambda p=0$, which holds true exactly in case $x \Psi(\lambda p)=0$. A similar observation can be applied concerning the multiplication by $x$ from the other side. One can easily show that these imply the existence of the desired $\mu$.

As a consequence of Step C, Lemma 3 and [12, Theorem 4.5], we conclude that for every $\alpha \in \Gamma$ there are an index $f(\alpha)$, a ring automorphism $h_{\alpha}: \mathbb{C} \rightarrow \mathbb{C}$ and an invertible matrix $A_{\alpha}$ such that the $\alpha$ th coordinate function of $\Phi, \Phi_{\alpha}$, is either of the form

$$
\Phi_{\alpha}(x)=A_{\alpha}\left[h_{\alpha}\left(x_{f(\alpha)}\right)\right] A_{\alpha}{ }^{-1} \quad \text { or } \quad \Phi_{\alpha}(x)=A_{\alpha}\left[h_{\alpha}\left(x_{f(\alpha)}\right)\right]^{t} A_{\alpha}{ }^{-1},
$$

where $h_{\alpha}\left(x_{f(\alpha)}\right)$ denotes the matrix obtained from $x_{f(\alpha)} \in C_{p}\left(H_{f(\alpha)}\right)$ by applying $h_{\alpha}$ for every entry of it.

Step E. $f: \Gamma \rightarrow \Gamma$ is injective, and every $h_{\alpha}$ is continuous except for a finite number of indexes.

To the injectivity of $f$ suppose $\alpha \neq \alpha^{\prime}$ and $f(\alpha)=f\left(\alpha^{\prime}\right)$. For the identity operator $\mathbf{1}_{\alpha}$ on $H_{\alpha}$ let us consider the element $\overline{\mathbf{1}_{\alpha}} \in l_{p}\left(C L_{p}\left(H_{\alpha}\right), c_{\alpha}, \Gamma\right)$. Since an isomorphism maps identities into each other, we arrive at a contradiction. As for the second statement, assume that there are infinitely many indexes for which the corresponding $h_{\alpha}$ is discontinuous. Let $\left(\alpha_{n}\right)$ be a sequence of such indexes. The discontinuous automorphisms of $\mathbb{C}$ are unbounded on every disc $[8$, Theorem 2 , p. 360] (cf. [1]). Thus there is a sequence of complex numbers $\left(\lambda_{\alpha_{n}}\right)$ such that

$$
\sum_{n} c_{f\left(\alpha_{n}\right)} \operatorname{dim} H_{f\left(\alpha_{n}\right)}\left|\lambda_{\alpha_{n}}\right|^{p}<\infty \quad \text { but } \quad \sum_{n} c_{\alpha_{n}} \operatorname{dim} H_{\alpha_{n}}\left|h_{\alpha_{n}}\left(\lambda_{\alpha_{n}}\right)\right|^{q}=\infty .
$$

From the convergence of this first series we infer

$$
\sum_{n} \overline{\lambda_{\alpha_{n}} \mathbf{1}_{f\left(\alpha_{n}\right)}} \in l_{p}\left(C L_{p}\left(H_{\alpha}\right), c_{\alpha}, \Gamma\right),
$$

while the other relation shows that using

$$
\left(\Phi\left(\sum_{k} \overline{\left.\lambda_{\alpha_{k}} \mathbf{1}_{f\left(\alpha_{k}\right)}\right)}\right)\right)_{\alpha_{n}}=\Phi_{\alpha_{n}}\left(\sum_{k} \overline{\lambda_{\alpha_{k}} \mathbf{1}_{f\left(\alpha_{k}\right)}}\right)=h_{\alpha_{n}}\left(\lambda_{\alpha_{n}}\right) \mathbf{1}_{\alpha_{n}},
$$

we would have $\Phi\left(\sum_{k} \overline{\left.\lambda_{\alpha_{k}} \mathbf{1}_{f\left(\alpha_{k}\right)}\right)} \notin l_{q}\left(C L_{q}\left(H_{\alpha}\right), c_{\alpha}, \Gamma\right)\right.$, which is an obvious contradiction.

In our last lemma we need the following notation. For $1 \leq r<\infty$ and a sequence $\left(w_{n}\right)$ of positive numbers let

$$
\begin{aligned}
& l_{r}(w)=\left\{\left(\lambda_{n}\right):\left(\lambda_{n}\right)\right. \text { is a sequence of complex numbers } \\
& \left.\qquad \text { with }\left(\sum_{n} w_{n}\left|\lambda_{n}\right|^{r}\right)^{1 / r}<\infty\right\} .
\end{aligned}
$$


Lemma 4. If $\left(c_{n}\right)$ and $\left(d_{n}\right)$ are sequences of positive numbers, then $l_{p}(c) \neq l_{q}(d)$.

Let $p<q$, and suppose, conversely, that

$$
\sum_{n} c_{n}\left|\lambda_{n}\right|^{p}<\infty \Longleftrightarrow \sum_{n} d_{n}\left|\lambda_{n}\right|^{q}<\infty
$$

or with $r=q / p>1$ that

$$
\sum_{n} c_{n}\left|\lambda_{n}\right|<\infty \Longleftrightarrow \sum_{n} d_{n}\left|\lambda_{n}\right|^{r}<\infty
$$

If $\left(u_{n}\right)=\left(\frac{c_{n}}{d_{n}{ }^{1 / r}}\right)$, then for every $\left(\theta_{n}\right) \in l_{r}$ we have $\left(\theta_{n} u_{n}\right) \in l_{1}$. An easy application of the principle of uniform boundedness shows that $\left(u_{n}\right) \in l_{s}$, where $s$ is the exponent conjugate to $r$. As a special case, it follows that

$$
\frac{c_{n}}{d_{n}{ }^{1 / r}} \longrightarrow 0
$$

Since the identity operator $I: l_{r}(d) \rightarrow l_{1}(c)$ is continuous, by the Banach theorem on the bounded inverse there is a positive constant $k$ such that

$$
\left(\sum_{n} d_{n}\left|\lambda_{n}\right|^{r}\right)^{1 / r} \leq k \sum_{n} c_{n}\left|\lambda_{n}\right|
$$

holds for every $\left(\lambda_{n}\right) \in l_{1}(c)$. But this implies that $\left(\frac{d_{n}^{1 / r}}{c_{n}}\right)$ is bounded, which contradicts the convergence above.

As for the rest of the proof, let $F \subset \Gamma$ be a (possibly empty) finite set such that for every $\alpha \in \Gamma \backslash F$, the function $h_{\alpha}$ is a continuous automorphism of $\mathbb{C}$. It is well known that there are exactly two such automorphisms, namely the identity and the conjugation [8, Lemma 1, p. 356], [1]. Let $\left(\alpha_{n}\right)$ be a sequence of pairwise distinct elements of $\Gamma \backslash F$. Taking Lemma 4 into account, assume that there is a sequence $\left(\lambda_{\alpha_{n}}\right)$ of complex numbers such that

$$
\sum_{n} c_{f\left(\alpha_{n}\right)} \operatorname{dim} H_{f\left(\alpha_{n}\right)}\left|\lambda_{\alpha_{n}}\right|^{p}=\infty \quad \text { but } \quad \sum_{n} c_{\alpha_{n}} \operatorname{dim} H_{\alpha_{n}}\left|\lambda_{\alpha_{n}}\right|^{q}<\infty .
$$

Then similarly to the argument in the proof of Step E above, one can prove that $\sum_{n} \overline{\lambda_{\alpha_{n}} \mathbf{1}_{\alpha_{n}}} \in l_{q}\left(C L_{q}\left(H_{\alpha}\right), c_{\alpha}, \Gamma\right)$ and that it does not belong to the range of $\Phi$, which is untenable. In the case that was not treated the proof may proceed in a similar way.

Remark. If we define $C_{\infty}(\mathcal{A})=C(\mathcal{A})$, then using almost the same argument one can prove the relevant statement for $p=\infty$ as well. We also remark that in case $\mathcal{A}$ is finite dimensional, the conclusion of our theorem fails to be true, which is a trivial consequence of the fact that in this case $C_{p}(\mathcal{A})=C_{q}(\mathcal{A})$.

To conclude the paper we note that it would be of some interest to investigate topological differences between these classes as well. 


\section{REFERENCES}

1. J. Aczél and J. Dhombres, Functional equations in several variables, Encyclopedia Math. Appl., vol. 31, Cambridge Univ. Press., Cambridge, 1989. MR 90h:39001

2. W. Ambrose, Structure theorems for a special class of Banach algebras, Trans. Amer. Math. Soc. 57 (1945), 364-386. MR 7:126c

3. B.H. Arnold, Rings of operators on vector spaces, Ann. of Math. 45 (1944), 24-49. MR 5:147c

4. F.F. Bonsall and J. Duncan, Complete normed algebras, Springer, New York, 1973. MR 54:11013

5. R.W. Cross, On the continuous linear image of a Banach space, J. Austral. Math. Soc. Ser. A 29 (1980), 219-234. MR 81f:47001

6. I.N. Herstein, Topics in ring theory, Univ. of Chicago Press, Chicago, IL, 1969. MR 42:6018

7. N. Jacobson and C. Rickart, Jordan homomorphisms of rings, Trans. Amer. Math. Soc. 69 (1950), 479-502. MR 12:387h

8. M. Kuczma, An introduction to the theory of functional equations and inequalities, PWN, Warszawa, 1985. MR 86i:39008

9. J. La Duke, On a certain generalization of $l_{p}$ spaces, Pacific J. Math. 35 (1970), 155-168. MR 43:896

10. L. Molnár, *-representations of the trace-class of an $H^{*}$-algebra, Proc. Amer. Math. Soc. 115 (1992), 167-170. MR 92i:46064

11., p-classes of an $H^{*}$-algebra and their representations, Acta Sci. Math. (Szeged) $\mathbf{5 8}$ (1993), 411-423. MR 95c:46081

12. M. Omladič and P. Šemrl, Additive mappings preserving operators of rank one, Linear Algebra Appl. 182 (1993), 239-256. MR 94f:47039

13. P.P. Saworotnow and J.C. Friedell, Trace-class for an arbitrary $H^{*}$-algebra, Proc. Amer. Math. Soc. 26 (1970), 95-100. MR 42:2304

14. P.P. Saworotnow, Trace-class and centralizers of an $H^{*}$-algebra, Proc. Amer. Math. Soc. 26 (1970), 101-104. MR 42:2305

15. P.P. Saworotnow and G.R. Giellis, Continuity and linearity of centralizers on a complemented algebra, Proc. Amer. Math. Soc. 31 (1972), 142-146. MR 44:5781

16. P.P. Saworotnow, Generalized positive definite functions and stationary processes, Prediction Theory and Harmonic Analysis (V. Mandrekar and H. Salehi, eds.), North-Holland, Amsterdam, 1983, pp. 329-343. MR 84j:60045

17. D Diagonalization of a self-adjoint operator acting on a Hilbert module, Internat. J. Math. Math. Sci. 8 (1985), 669-675. MR 87f:46084

18. P. Šemrl, Additive derivations of some operator algebras, Illinois J. Math. 35 (1991), 234-240. MR 92b: 47068

19. _ Ring derivations on standard operator algebras, J. Funct. Anal. 112 (1993), 318-324. MR 94h: 47084

20. _ Isomorphisms of standard operator algebras, Proc. Amer. Math. Soc. 123 (1995), 1851-1855. MR 95g:47066

21. J.F. Smith, The p-classes of an $H^{*}$-algebra, Pacific J. Math. 42 (1972), 777-793. MR 48:879

22. P.K. Wong, The p-class in a dual $B^{*}$-algebra, Trans. Amer. Math. Soc. 200 (1974), 355-368. MR 50:10837

Institute of Mathematics, Lajos Kossuth University, 4010 Debrecen, P.O. Box 12, HUNGARY

E-mail address: molnarl@math.klte.hu 